\title{
Mapping the methane on Mars
}

\author{
S. Fonti ${ }^{1}$ and G. A. Marzo ${ }^{2}$ \\ 1 Department of Physics, Università del Salento, via Arnesano C.P. 193, 73100 Lecce, Italy \\ e-mail: sergio.fonti@le.infn.it \\ 2 NASA Ames Research Center, Space Science and Astrobiology Division, MS 245-3, Moffett Field, CA 94035-1000, USA \\ e-mail: giuseppe.marzo@nasa.gov
}

Received 25 August 2009 / Accepted 22 December 2009

\section{ABSTRACT}

Context. In the past few years several authors have reported detection of a very small amount of methane in the martian atmosphere. Suggested hypotheses for this methane production can be linked either to geological or biological activities, and in both cases our perspective on the planet evolution would require a major revision.

Aims. It has been suggested that methane is heterogeneously distributed in the atmosphere, but its temporal and spatial variability is still unknown. Our goal is to describe the distribution of the methane concentration in the martian atmosphere and its temporal evolution, providing insight into its sources and constraining its sinks.

Methods. Using the extensive data set of the thermal emission spectrometer onboard Mars Global Surveyor and a statistical clustering technique, here we tracked the gas evolution over three martian years using the $v_{4}$ Q-branch methane band at $1306 \mathrm{~cm}^{-1}$.

Results. We identify three localized sources, corresponding to Arabia Terrae, well known for the high content of underground water, and to the two main volcano provinces, Tharsis and Elysium. Our analysis suggests a seasonal cycle of the methane distribution, as well as interannual variations. This discovery potentially constrains the lifetime of the methane molecule in the martian atmosphere to $<1 \mathrm{yr}$, suggesting that there is a process able to remove the methane from the atmosphere much more efficiently than photochemistry ( $\sim 350 \mathrm{yr})$. High concentrations of methane are found in the warmest seasons, when relatively high energy could trigger volatile release, which is related either to geological processes, or outbreaks of biological activity. Our results, obtained with independent data in a new spectral range, are quantitatively consistent with previous estimates of methane concentration, building confidence that there is methane on Mars.

Key words. planets and satellites: individual: Mars - infrared: planetary systems - techniques: spectroscopic

\section{Introduction}

The question of whether there is methane in the martian atmosphere has generated a wide debate about its likely biological or geological origin (Summers et al. 2002; Pellenbarg et al. 2003; Onstott et al. 2006; Prieto-Ballesteros et al. 2006; Atreya et al. 2007; Chastain \& Chevrier 2007). In either case, this would change our perspective on the planet's evolution (Dohm et al. 2008).

Ground-based spectroscopic measurements (Krasnopolsky et al. 1997, 2004) were the basis of the first claim that there is methane on Mars. The main problem with these measurements is accounting precisely for the contribution of the methane in the terrestrial atmosphere, so that emphasis was put on detecting the $3018 \mathrm{~cm}^{-1} v_{3}$ Q-branch methane band using the planetary Fourier spectrometer (PFS) onboard ESA Mars Express (Formisano et al. 2004). Such a detection was reported for a few different orbits in several areas scattered over the whole surface of Mars, with a footprint ranging from about ten to some hundred kilometres and a spectral resolution of $1.3 \mathrm{~cm}^{-1}$. Very recently a spatially resolved detection of methane, obtained using ground-based high-resolution spectral measurements of the R0 and R1 methane lines of the Q-branch at 3029 and $3039 \mathrm{~cm}^{-1}$ has been reported over Nili Fossae (Mumma et al. 2009). All these detections were made in the spectral region of the strongest methane feature ( $v_{3}$ Q-branch), and the confidence about the presence of methane in the atmosphere of Mars would increase greatly if it could also be detected using its second strongest band, the $v_{4}$ Q-branch at $1306 \mathrm{~cm}^{-1}$.
For this purpose, we decided to use the data collected in this spectral region by the thermal emission spectrometer (TES) onboard Mars Global Surveyor (MGS) (Christensen et al. 2001), which provides full spatial coverage of Mars over about three martian years (MYs) (Clancy et al. 2000), i.e. the second half of MY24, MY25, MY26, and the first half of MY27. Even if its relatively low spectral resolution (two modes at 12.5 and $6.25 \mathrm{~cm}^{-1}$ with spectral sampling at $10 \mathrm{~cm}^{-1}$ and $5 \mathrm{~cm}^{-1}$, respectively) could not, in principle, be useful for detecting such a narrow feature, we used both an experimental and a computational approach to verify that the $1306 \mathrm{~cm}^{-1}$ methane band (hereafter the methane band) is detectable by averaging a few thousand spectra, thanks to TES relatively high noise equivalent spectral radiance (NESR) (Christensen et al. 1998, 2001).

In this paper we first show that resolutions, such those of TES $\left(6.25\right.$ and $\left.12.5 \mathrm{~cm}^{-1}\right)$, could be sufficient to detect the methane feature at $1306 \mathrm{~cm}^{-1}$. Then we use a statistical cluster analysis technique by Marzo et al. $(2006,2008)$ to group the TES spectra by the presence of such feature and show their spatial and temporal distribution. Finally we discuss our findings and draw some conclusions.

\section{Methane spectrum at low resolution}

To characterize the differences in the $1306 \mathrm{~cm}^{-1}$ methane band going from a relatively high- $\left(0.2 \mathrm{~cm}^{-1}\right)$ to low-resolution spectrum $\left(12.5 \mathrm{~cm}^{-1}\right)$, we measured the trasmittance of a pure methane sample contained in a $1.6 \mathrm{~mm}$ thick sealed cell at 
Table 1. Estimated global amount of methane.

\begin{tabular}{ccccccc}
\hline \hline Temporal slice & Resolution & Number of Spectra & Spectra in MC & Methane index & Detection limit (ppbv) & Methane content (ppbv) \\
\hline MY24 Ls180 & $12.5 \mathrm{~cm}^{-1}$ & 516701 & $213522(41 \%)$ & 0.7512 & 2 & $33 \pm 9$ \\
MY24 Ls270 & $12.5 \mathrm{~cm}^{-1}$ & 159201 & $28428(18 \%)$ & 0.1374 & 6 & $6 \pm 2$ \\
MY25 Ls0 & $12.5 \mathrm{~cm}^{-1}$ & 359717 & $144898(40 \%)$ & 0.3715 & 3 & $17 \pm 5$ \\
MY25 Ls90 & $12.5 \mathrm{~cm}^{-1}$ & 442296 & $136755(30 \%)$ & 0.3165 & 3 & $14 \pm 4$ \\
\hline MY25 Ls180 & $6.25 \mathrm{~cm}^{-1}$ & 68901 & $18495(27 \%)$ & 0.4034 & 4 & $18 \pm 7$ \\
MY25 Ls270 & $6.25 \mathrm{~cm}^{-1}$ & 143840 & $43438(30 \%)$ & 0.1223 & 3 & $5 \pm 2$ \\
MY26 Ls0 & $6.25 \mathrm{~cm}^{-1}$ & 69349 & $14003(20 \%)$ & 0.2344 & 4 & $\ldots \pm 4$ \\
MY26 Ls90 & $6.25 \mathrm{~cm}^{-1}$ & $\ldots$ & $\ldots$ & $\ldots$ & $\ldots$ \\
\hline MY26 Ls180 & $12.5 \mathrm{~cm}^{-1}$ & 507365 & $200508(40 \%)$ & 0.6696 & 2 & $5 \pm 8$ \\
MY26 Ls270 & $12.5 \mathrm{~cm}^{-1}$ & 320180 & $45961(14 \%)$ & 0.1124 & 5 & $5 \pm 1$ \\
MY27 Ls0 & $12.5 \mathrm{~cm}^{-1}$ & 197327 & $42677(22 \%)$ & 0.2114 & 5 & $9 \pm 3$ \\
MY27 Ls90 & $12.5 \mathrm{~cm}^{-1}$ & 115194 & $38041(33 \%)$ & 0.6203 & 5 & $28 \pm 8$ \\
\hline
\end{tabular}

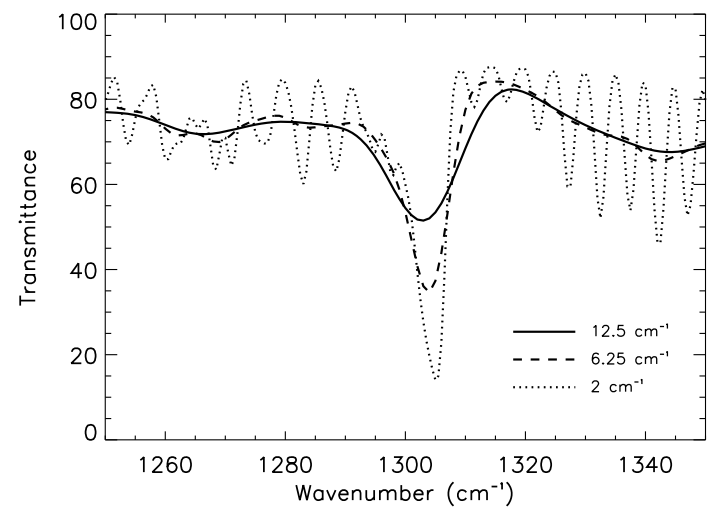

Fig. 1. Laboratory transmittance spectra of a methane cell taken at different resolutions, shown in the wavelength interval of interest.

standard atmospheric pressure and temperature. The spectra were recorded using a Perkin Elmer Spectrum 2000 Fourier Spectrometer and are shown in Fig. 1. As seen, the spectral contrast is only reduced by a factor 1.5 going from 2 to $6.25 \mathrm{~cm}^{-1}$, and of a factor 2.5 when going from 2 to $12.5 \mathrm{~cm}^{-1}$. In addition, the spectral contrast of the methane feature at a resolution of $2 \mathrm{~cm}^{-1}$ is more than 0.9 of that collected at a resolution of $0.2 \mathrm{~cm}^{-1}$, which is not reported in Fig. 1 for the sake of clarity. From the spectra shown in Fig. 1, it is clear that it would be highly desirable to use only the TES data collected at $6.25 \mathrm{~cm}^{-1}$ resolution, but they are a small fraction of the data collected at $12.5 \mathrm{~cm}^{-1}$ resolution.

In this respect we investigated the lowest amount of methane detectable by TES by applying the calculation of Maguire (1977) for the case of IRIS. The IRIS data set consists of 1747 infrared spectra (from 200 to $2000 \mathrm{~cm}^{-1}$ ) with a spectral resolution of $2.4 \mathrm{~cm}^{-1}$ scattered throughout the entire planet. Given the average NESR of $5.0 \times 10^{-8} \mathrm{~W}^{-1} \mathrm{~cm}^{-2} \mathrm{str}^{-1} \mathrm{~cm}^{-1}$ at $\sim 270 \mathrm{~K}$ for a single spectrum, Maguire estimated a maximum detectable average mixing ratio of 20 ppbv. The same calculation can be applied to TES, given its NESR and spectral resolution, for evaluating how many spectra must be averaged to obtain the detection of methane at $10 \mathrm{ppbv}$ level, which is the mean mixing ratio claimed by Krasnopolsky et al. (2004) and Formisano et al. (2004). Since the average NESR of TES is $1.2 \times 10^{-8} \mathrm{~W}^{-1} \mathrm{~cm}^{-2} \mathrm{str}^{-1} \mathrm{~cm}^{-1}$ at $270 \mathrm{~K}$ for a single spectrum (Christensen et al. 1998), we obtain 3000 spectra for the resolution of $6.25 \mathrm{~cm}^{-1}$ and 11000 spectra for $12.5 \mathrm{~cm}^{-1}$, well within the capability of the extensive TES database.

\section{Method of analysis}

We selected nearly 3000000 TES spectra covering the region between $60^{\circ} \mathrm{S}$ and $60^{\circ} \mathrm{N}$ in latitude. Temporally they are centred at each equinox and solstice for each of the three MY considered. In addition they were collected during the warmest part of the martian day and in nadir configuration, in order to have constant spatial resolution $(\sim 3 \mathrm{~km})$ and high radiance. During MY24/25 and 26/27, TES operated mainly in the low-resolution mode $\left(12.5 \mathrm{~cm}^{-1}\right)$ and in high-resolution mode $\left(6.25 \mathrm{~cm}^{-1}\right)$ in MY25/26. This selection should provide 12 spatial/temporal slices, but the amount of usable spectra selected in the summer (solar songitude, Ls $90^{\circ}$ ) of MY26 was too low for considering this slice statistically reliable, so we decided to perform our analysis only on 11 slices. In all of them, the number of averaged spectra was well in excess of the limit evaluated above, ranging between 14000 and 43000 for the resolution of $6.25 \mathrm{~cm}^{-1}$ and between 28000 and 213000 for $12.5 \mathrm{~cm}^{-1}$ (see Table 1).

We explored each spatial/temporal slice by using a cluster analysis approach (Marzo et al. 2006, 2008, 2009), which allowed us to selectively average tens of thousands of spectra, corresponding to a minimum quantity of detectable $\mathrm{CH}_{4}$ ranging between 2 and 6 ppbv. Our cluster analysis is based on a partitioning technique that is a statistical multivariate framework that iteratively groups spectra by minimizing the dimension of the clusters and, simultaneously, maximizing their distance in the data space. The main consequence of this approach is that the final cluster configuration does not depend on the random noise of measurements, while the technique is very sensitive to systematic errors, such as instrumental effects (Marzo et al. 2006, 2008). This technique has been extensively tested using TES data and has been proven fully effective, producing results in very close agreement with those obtained using traditional data sorting procedures. In particular we have shown the ability of cluster analysis to successfully retrieve different surface mineralogies (cf. Fig. 1 in Marzo et al. 2006; and Fig. 2 in Marzo et al. 2008), as well as of identifying distinct amount of $\mathrm{CO}_{2}$ and $\mathrm{H}_{2} \mathrm{O}$, aerosol, and water ice content in the martian atmosphere (cf. Fig. 2 in Marzo et al. 2008).

The cluster analysis algorithms were applied to the narrow spectral region where the methane band is expected, and no further selection or pre-processing has been applied to the data. No alterations to the original spectra, or to the spectral range result after application of the algorithm, and the output is the association of each spectrum to a specific cluster. This procedure has proved to be equivalent to the evaluation of the band depth in each spectrum (Marzo et al. 2008). The first application of the 
algorithm provides a large cluster, including almost the entire data set, and a variable number of small clusters, including peculiar martian spectra or spurious data affected by distinct instrumental effects (e.g. very cold spectra, spiky measurements, etc.), which were obviously excluded from further analysis. The second application always provided two clusters, grouped according to the different values in the methane band spectral region. On average, approximately one third of the spectra in each slice belongs to the cluster that presents the methane band (hereafter $\mathrm{MC}$, methane cluster), even if these spectra show a strong spatial variability.

At the end of the clustering, we have three quantities for each temporal slice: a) the total number of spectra (reported in the third column of Table 1; b) the average of all the spectra included in MC (reported, also in percentage, in the fourth column of Table 1); c) the number of remaining spectra, not included in the MC. To estimate the global amount of methane present in each temporal slice we account for both the number of spectra present in the associated MC and the methane band depth in the corresponding average spectrum. After several attempts, we have found that the best way to accomplish such a difficult task is to define the methane index (MI), reported in the fifth column of Table 1. Such MI is basically the methane band depth, evaluated in the ratio between the average spectra a) and c). The values relative to the spectra acquired in higher resolution mode have been properly scaled to be comparable with those taken at lower resolution. At the end of this evaluation, we can only state the relative amount of methane in each temporal slice; in other words, we know that in the summer (Ls 90 $)$ of MY25 the amount of methane we detect is approximately half that observed in Summer of MY27, but we still need a reliable normalization procedure. For this purpose, we used the detection limits discussed in the last paragraph of the previous section and listed in the sixth column of Table 1 . We assumed that the methane is at its detection limit in the slice with the lowest methane amount, namely that corresponds to the winter (Ls 270 $)$ of MY26 and then we scaled all other values accordingly. Therefore, strictly speaking, our evaluation of the methane content in each temporal slice has to be considered as a lower limit, since the amount of methane observed in the winter of MY26 is certainly above (although most probably only slightly above) its detection limit, otherwise we would not be able to detect it.

\section{Results and discussions}

In the last column of Table 1, we list the global quantity of methane at the time of each observation. The uncertainties reported in Table 1 are mostly due to the calibration of the detection limit (Maguire 1977) and to other possible misjudgment sources, such as the influence of local topography. The error associated with our statistical analysis is much lower: about $3 \%$ for the lower resolution data and about $14 \%$ for the higher resolution spectra. In the maps of Fig. 2, we show the spatial distribution of methane obtained by dividing each slice into geographic cells of $10^{\circ}$ in latitude and $10^{\circ}$ in longitude. The quantity of methane present in each cell was assumed to be proportional to the normalized number of spectra belonging to the MC present in each cell, compared to the normalized number of MC spectra of the corresponding slice. The grid is sufficient to illustrate the spatial variability of the methane content in the martian atmosphere and, at the same time, is wide enough to smooth the influence of local topography. It is obviously impossible to evaluate the amount of methane present in each geographic cell using the complex procedure described at the end of the previous section for the entire planet, since the number of spectra within each cell is usually well below what are necessary for the detection of methane, even if present in relatively large quantities (see discussion about the detection limits at the end of Sect. 2). Therefore we assume that the quantity of methane present in each cell is proportional to the normalized number of spectra belonging to the MC present in each cell, compared to the normalized number of MC spectra of the corresponding temporal slice. As an example, if we evaluate $30 \mathrm{ppbv}$ in a given temporal slice, for which the percentage of methane spectra is $40 \%$ and if we find $20 \%$ or $80 \%$ of the methane spectra in a given geographic cell of that slice, we conclude that the amount of methane is 15 or 60 ppbv, respectively. We checked that the reliability of the method is acceptable, decreasing sharply when the total number of spectra in a given geographic cell is particularly low. We simply assumed that no methane is present in the very few cases when such conditions occurred.

We have considered the possibility of a different attribution of the observed band centred at $1306 \mathrm{~cm}^{-1}$. We performed a thorough investigation studying each possible contribution to the band from the known solid and gaseous species reported on Mars. For this we used a MODTRAN radiative transfer code, including a complete description of the atmosphere and the surface of Mars. The gaseous species included are $\mathrm{CO}_{2}$ (and its major isotopes), $\mathrm{H}_{2} \mathrm{O}$ (water vapor), $\mathrm{O}_{2}$ (oxygen), $\mathrm{CO}$ (carbon oxide), and $\mathrm{CH}_{4}$ (methane), while water ice and aerosol opacity, together with the surface end-members were derived by Bandfield $\&$ Smith (2003). Estimates of the surface temperatures and the atmospheric temperature profiles were retrieved by averaging the profiles provided by the TES measurements. Each parameter involved in the analysis was varied, observing its effects in the spectral region of interest (from 1230 to $1400 \mathrm{~cm}^{-1}$ ). From these simulations, a possible influence on the observed $1306 \mathrm{~cm}^{-1}$ band by anything else other than methane has not been observed.

In Fig. 3 we compare two selected MC averages, one for each of the two different resolutions, with a set of synthetic spectra computed for martian conditions. As can be seen, the spectral region is not significantly influenced by solid materials, which is not surprising, because the solid materials do not possess such narrow features around $1300 \mathrm{~cm}^{-1}$ (Salisbury et al. 1991; Ruff et al. 1997). Essentially for the same reason, the aerosol is transparent in most of this spectral range, in particular around $1300 \mathrm{~cm}^{-1}$ (Bandfield \& Smith 2003). The influence of temperature profile and surface temperature is not relevant in this spectral region, since their effects are negligible with respect to the mixing ratio variation of the gaseous $\mathrm{H}_{2} \mathrm{O}, \mathrm{CO}_{2}$, and $\mathrm{CH}_{4}$, which are the only three atmospheric components actually affecting the 1230 to $1400 \mathrm{~cm}^{-1}$ spectral region. Even if other minor species, such as $\mathrm{O}_{2}$ and $\mathrm{CO}$, might, in principle, contribute to the resulting spectrum, we have verified that their only effect is a negligible vertical offset, constant in the whole spectral interval.

From inspection of Fig. 3 we see that the feature, present at $1306 \mathrm{~cm}^{-1}$ in the MC averages and marked with a dashed line, is evident only in the methane spectrum and cannot be easily ascribed to any of the other spectra shown. It is interesting to note that the other features present in the lower resolution MC spectrum are correlated very well to the spectral features of $\mathrm{CO}_{2}$ (dotted lines), while the contribution of $\mathrm{H}_{2} \mathrm{O}$ cannot be clearly assessed at this spectral resolution. On the contrary, the influence of water vapor is apparent in the higher resolution $\mathrm{MC}$ spectrum (top right panel, Fig. 3), where the MC spectral features are, in most cases, well correlated to features present in both $\mathrm{CO}_{2}$ and $\mathrm{H}_{2} \mathrm{O}$ spectra. 


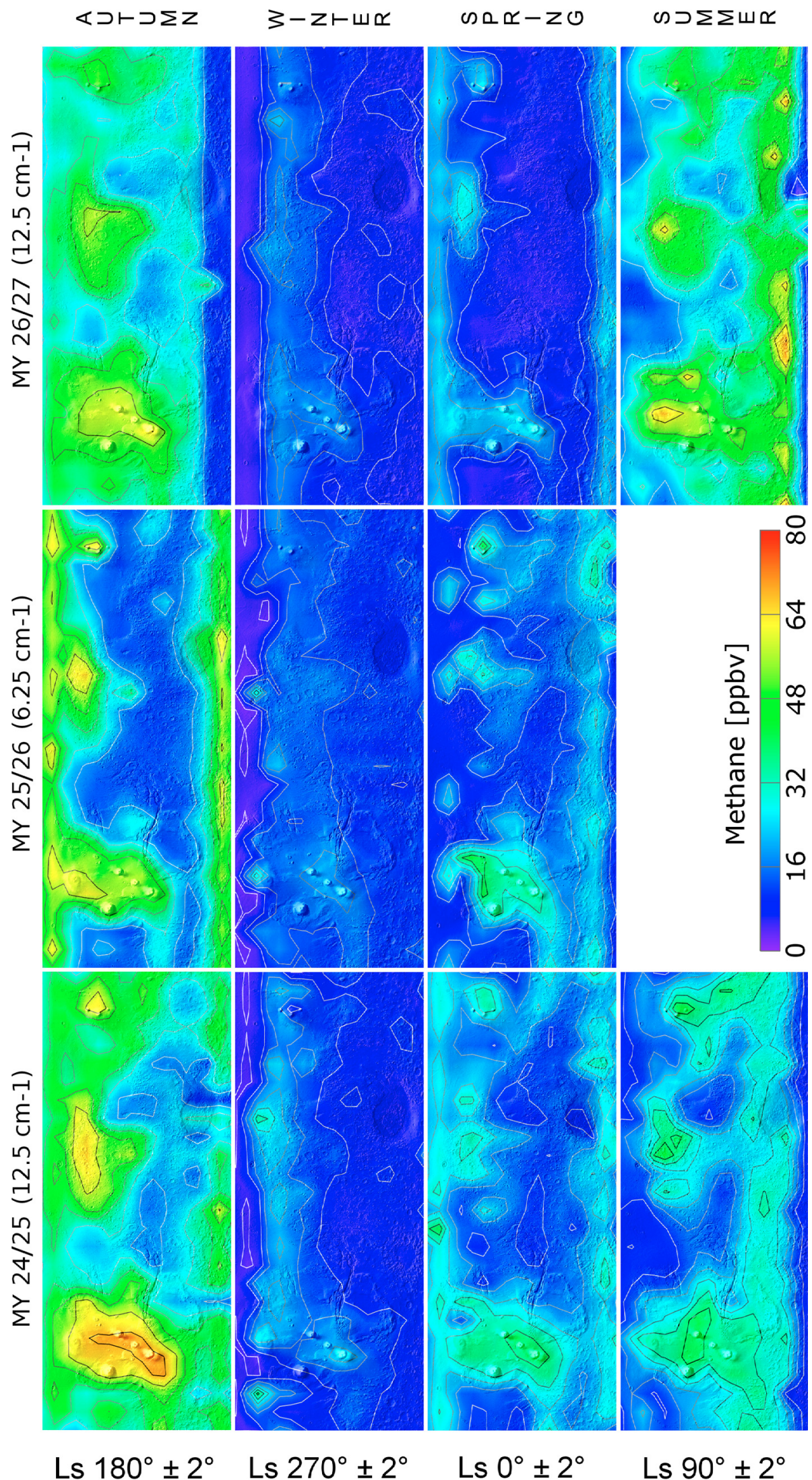

Fig. 2. Summary of the spatial and temporal distribution of methane, reported on a martian map, centred, in solar longitude (Ls), at each equinox and solstice $\left(0^{\circ}, 90^{\circ}, 180^{\circ}\right.$, and $270^{\circ} \pm 2^{\circ}$ ), for each of the three martian years considered in the present study. Each map is limited in latitude between $60^{\circ} \mathrm{S}$ and $60^{\circ} \mathrm{N}$. The colour code used in the figure and described in the colour bar is representative of the relative number of spectra, belonging to the methane cluster present in each spatial cell. The map corresponding to the summer of MY26 has not been calculated, because of the lack of usable data available. On the right side we report, for convenience, the name of the martian seasons referred to the northern hemisphere.
Our simulations illustrate that the possibility of the attribution of the feature at $1306 \mathrm{~cm}^{-1}$ to anything else than methane is unlikely, however, to further evaluate the possible contamination of water vapour, we compared the temporal evolution of the intensity of the $1306 \mathrm{~cm}^{-1}$ band with the water vapour distribution obtained by Smith (2004) using the same TES data. The comparison is shown in Fig. 4 and the opposite behaviour is apparent, including a temporal phase shift of about $90^{\circ}$ in Ls. The main conclusion of this analysis is that the band at $1306 \mathrm{~cm}^{-1}$ in the martian spectra definitely supports the hypothesis of the 

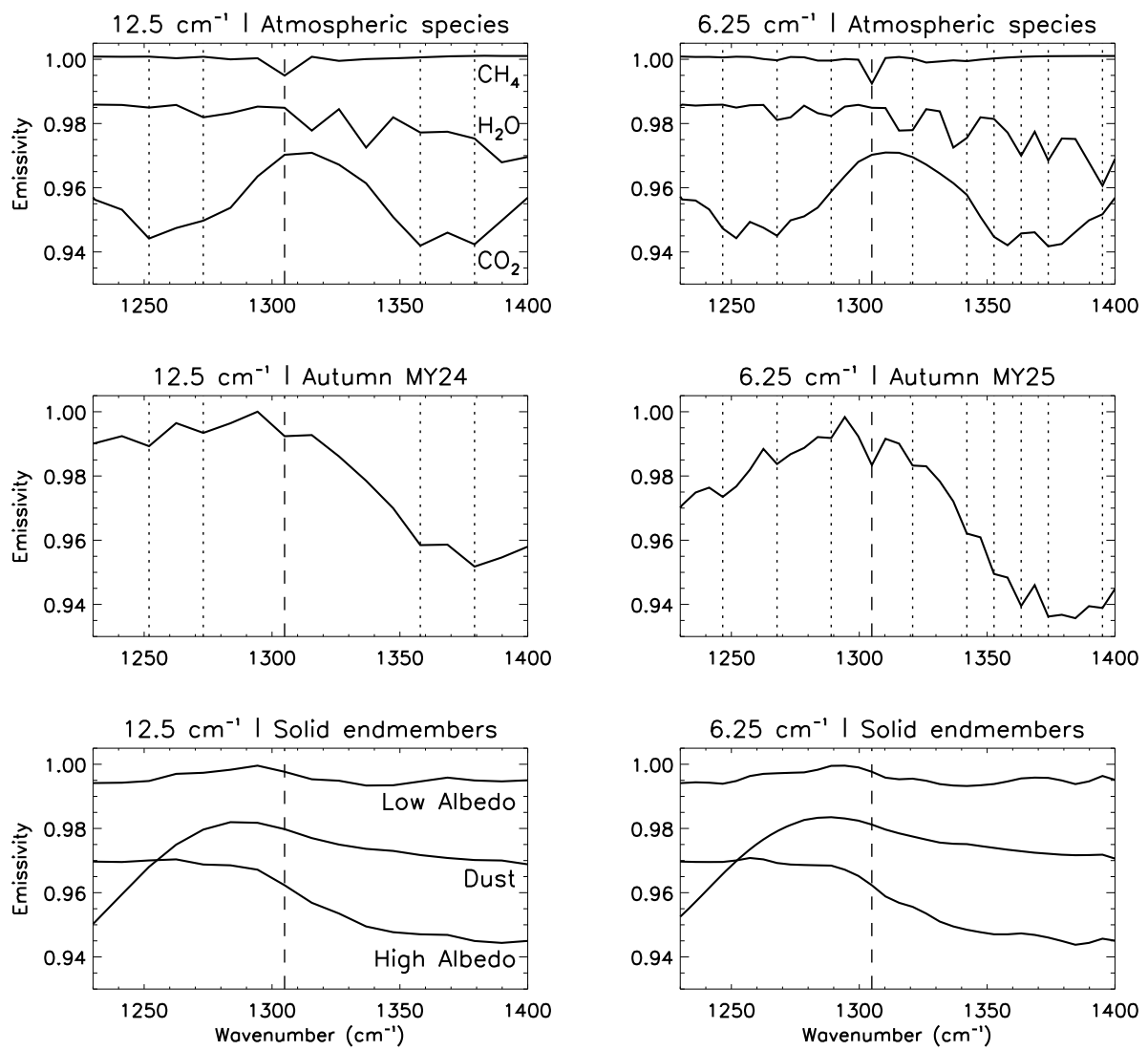

Fig. 3. Comparison of a set of synthetic spectra at TES resolutions with the observed average spectra corresponding to two methane clusters (middle panels). In the top panels $\mathrm{CO}_{2}$ (offset by -0.025$), \mathrm{H}_{2} \mathrm{O}$ (300 ppmv, offset by -0.015$)$, and $\mathrm{CH}_{4}(60 \mathrm{ppbv})$, computed in martian conditions, are reported. In the bottom panels the solid endmembers relative to low-albedo and high-albedo surfaces (offset by -0.025) are shown, along with the opacity-derived emissivity of aerosol dust (arbitrarily scaled).

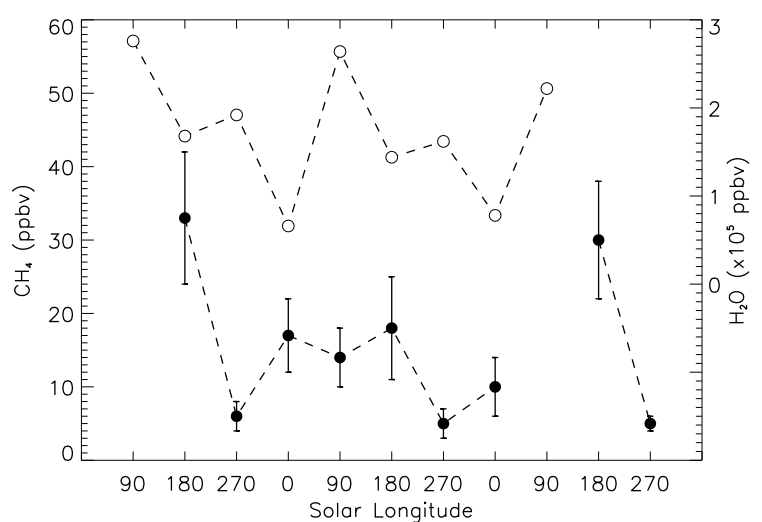

Fig. 4. Comparison between the temporal trend of the methane global content, derived from the values reported in the last column of Table 1 (black dots), and the water vapour abundance, as derived by Fig. 5 of Smith (2004), using the same TES data, in the same period of time (white dots).

presence of a tiny, but significant, amount of methane in the martian atmosphere.

The spatial distribution of methane is far from uniform with some regions of higher concentration and, at the same time, locations where the concentration of methane is very low, if not zero. We can underline that in each of the panels of Fig. 2 there are three broad regions where the methane amount is systematically higher (Tharsis, Arabia Terrae, and Elysium), and the concentration of methane seems to decrease moving from west (Tharsis) to east (Elysium). A similar decrease in the methane distribution has been reported using PFS data (Formisano et al. 2004; Geminale et al. 2008). Moreover, high concentrations of methane have been reported in Nili Fossae (Mumma et al. 2009), a province of Arabia Terrae. A higher concentration of methane around Elysium and Tharsis could be evidence of recent geological and hydrological activity in this martian region, where residual geothermal flux could still be active (Dohm et al. 2008). Magmatism, hydrothermal activity, and biological activity, sustained by these heat sources, are consistent with methane release (Krasnopolsky 2006; Onstott et al. 2006; Prieto-Ballesteros et al. 2006; Atreya et al. 2007). The northern and northeastern regions of Arabia Terrae have been instead associated with an extensive subsurface deposit of permafrost (Boynton et al. 2007), while the corresponding surface is enriched in hydrated silicates (Mustard et al. 2008) and other water-related minerals such as carbonates (Ehlmann et al. 2008). Hydration is a required condition for both the hydrogeochemical and the biogenic methane source hypotheses (Summers et al. 2002; Onstott et al. 2006; Atreya et al. 2007).

Coming to the temporal evolution of the methane abundance, Fig. 2 shows that the spatial distribution varies considerably, not only following a seasonal cycle but also from one year to the next. In this respect we wish to point out that the data displayed in Fig. 2 are not homogeneous, since during MY25/26, TES recorded almost exclusively spectra at high spectral resolution, while in MY24/25 and 26/27 the data have been collected in the low-resolution mode. Nevertheless, a definite decrease is quite evident in the methane abundance between MY24/25 and MY26/27, with a significant exception in summer. As far as the seasonal cycle is concerned, the maps clearly suggest a trend with the maximum relative abundance of methane in the northern autumn, a sharp decrease in winter, followed by a slight increase in spring, and a further increase in summer. In addition to the spatial distribution of methane, even more interesting is the spatial variation linked to the seasonal cycle. In northern autumn we see a spatial distribution extending to most of the planet with 
a higher abundance at both extremes of our latitude interval. The reduced amount of methane detected in winter is almost exclusively concentrated roughly between $40^{\circ}$ and $50^{\circ} \mathrm{N}$. In spring the spatial distribution is very similar to winter, but the overall relative abundance of methane is definitely higher. The spatial distribution in summer is similar, even if lower, to that in autumn, but, in this season, the highest spatial methane abundance is located between $40^{\circ}$ and $50^{\circ} \mathrm{S}$.

The temporal variations of the methane concentration rise questions not only concerning the possible methane sources but also about its sinks. An annual cycle, such as observed in Fig. 2, is consistent with a methane lifetime of 0.6 years (Mumma et al. 2009), which is much shorter than $~ 350$ years as previously suggested based on photochemical processes (Wong et al. 2003; Krasnopolsky et al. 2004). Strong oxidizers can be more effective than photochemistry in rapidly removing methane from the atmosphere (McDonald et al. 1998; Benner et al. 2000), and aeolian processes can favour the atmospheric introduction of oxidants (Delory et al. 2006; Atreya et al. 2007), such as $\mathrm{H}_{2} \mathrm{O}_{2}$ (Atreya et al. 2007) or the recently detected $\mathrm{XCl}_{4}$ (perchlorate) by the Phoenix lander (Hecht et al. 2009).

A direct quantitative comparison between our findings with the results of other authors (Formisano et al. 2004; Krasnopolsky et al. 2004; Mumma et al. 2009) reveals that, even if the temporal and the spatial scales are quite different, the global and regional concentrations of methane are strongly consistent with ours. In particular, it is interesting to compare our findings with the results recently published by Mumma et al. (2009). Even if the temporal match is not perfect (March 2003 vs. May 2003, a difference of $30^{\circ} \mathrm{Ls}$ ), the spatial distribution of methane is remarkably similar in the same region.

\section{Conclusions}

The conclusions that can be drawn from the results presented in the present paper can be summarized in the following list:

1. For the first time, methane has been pursued using the $v_{4}$ Q-branch band. In our view this is an unavoidable step for further confirming the real presence of such gas in the martian atmosphere, definitely ruling out any possible bias related to the use of the $v_{3}$ Q-branch band, the only one considered so far.

2. For the first time, after the detection of Formisano et al. (2004), we used the data of an orbiting spectrometer, proving that its low spectral resolution was not inadequate for this type of analysis. As anticipated in the introduction, the use of the huge MGS/TES database has allowed us to trace the spatial and temporal distribution of methane in the martian atmosphere with unprecedented accuracy and completeness. The results, validated by the simulations shown in Fig. 3, have been summarized in Fig. 2 and Table 1, showing clearly a) the presence of at least three localized sources (Tharsis, Arabia Terrae, and Elysium); b) temporal variations on time spans longer than one MY, as inferred by the different spatial distributions for corresponding seasons in MY24/25 and MY26/27; c) a seasonal cycle.

3. The annual cycle shown here could set an upper limit, $<1$ year, for the methane lifetime in the martian atmosphere. This would imply much more efficient mechanisms than photochemistry in removing methane from the atmosphere. These results provide the community with unprecedented insight into constraining the origin and the significance of the martian methane.
4. While our results cannot reveal the possible sources or sinks of the methane, it is evident that the highest concentrations are associated with the warmest seasons, and locations where favourable geological conditions, such as residual geothermal activity (Tharsis and Elysium) and strong hydration (Arabia Terrae), are expected on Mars. The higher energy available in these seasons could trigger both volatile release, which is related to geological processes, or outbreaks of biological activity.

5. Our estimates of the amount of methane in the martian atmosphere, inferred with the method described by Maguire (1977) and conveniently scaled to the characteristics of the MGS/TES spectrometer, are fully compatible with all the values given in the literature so far. However, the strong variability of such quantity, clearly shown in Fig. 2, definitely suggests that a proper comparison can only be made between spatially and temporally correlated data.

Acknowledgements. Part of this research was supported by an appointment to the NASA Postdoctoral Program at the Ames Research Center, administered by Oak Ridge Associated Universities through a contract with NASA. The authors warmly thank Armando Blanco, Ted Roush, Kevin Zahnle, Robert Haberle, and Geronimo Villanueva for constructive discussions and Therese Encrenaz for her very useful review.

\section{References}

Atreya, S. K., Mahaffy, P. R., \& Wong, A.-S. 2007, Planet. Space Sci., 55, 358 Bandfield, J. L., \& Smith, M. D. 2003, Icarus, 161, 47

Benner, S. A., Devine, K. G., Matveeva, L. N., \& Powell, D. H. 2000, Proceedings of the National Academy of Science, 97, 2425

Boynton, W. V., Taylor, G. J., Evans, L. G., et al. 2007, J. Geophys. Res. (Planets), 112, E12S99

Chastain, B. K., \& Chevrier, V. 2007, Planet. Space Sci., 55, 1246

Christensen, P. R., Anderson, D. L., Chase, S. C., et al. 1998, Science, 279, 1692

Christensen, P. R., Bandfield, J. L., Hamilton, V. E., et al. 2001, J. Geophys. Res., 106,23823

Clancy, R. T., Sandor, B. J., Wolff, M. J., et al. 2000, J. Geophys. Res., 105, 9553 Delory, G. T., Farrell, W. M., Atreya, S. K., et al. 2006, Astrobiology, 6, 451

Dohm, J. M., Anderson, R. C., Barlow, N. G., et al. 2008, Planet. Space Sci., 56, 985

Ehlmann, B. L., Mustard, J. F., Murchie, S. L., et al. 2008, Science, 322, 1828

Formisano, V., Atreya, S., Encrenaz, T., Ignatiev, N., \& Giuranna, M. 2004, Science, 306, 1758

Geminale, A., Formisano, V., \& Giuranna, M. 2008, Planet. Space Sci., 56, 1194 Hecht, M. H., Kounaves, S. P., Quinn, R. C., et al. 2009, Science, 325, 64 Krasnopolsky, V. A. 2006, Icarus, 180, 359

Krasnopolsky, V. A., Bjoraker, G. L., Mumma, M. J., \& Jennings, D. E. 1997, J. Geophys. Res., 102, 6525

Krasnopolsky, V. A., Maillard, J. P., \& Owen, T. C. 2004, Icarus, 172, 537 Maguire, W. C. 1977, Icarus, 32, 85

Marzo, G. A., Roush, T. L., Blanco, A., Fonti, S., \& Orofino, V. 2006, J. Geophys. Res. (Planets), 111, E03002

Marzo, G. A., Roush, T. L., Blanco, A., Fonti, S., \& Orofino, V. 2008, J. Geophys. Res. (Planets), 113, E12009

Marzo, G. A., Roush, T. L., \& Hogan, R. C. 2009, J. Geophys. Res. (Planets), 114, E08001

McDonald, G. D., de Vanssay, E., \& Buckley, J. R. 1998, Icarus, 132, 170

Mumma, M. J., Villanueva, G. L., Novak, R. E., et al. 2009, Science, 323, 1041

Mustard, J. F., Murchie, S. L., Pelkey, S. M., et al. 2008, Nature, 454, 305

Onstott, T. C., McGown, D., Kessler, J., et al. 2006, Astrobiology, 6, 377

Pellenbarg, R. E., Max, M. D., \& Clifford, S. M. 2003, J. Geophys. Res. (Planets), 108, 8042

Prieto-Ballesteros, O., Kargel, J. S., Fairén, A. G., et al. 2006, Geology, 34, 149

Ruff, S. W., Christensen, P. R., Barbera, P. W., \& Anderson, D. L. 1997, J. Geophys. Res., 102, 14899

Salisbury, J. W., D’Aria, D. M., \& Jarosewich, E. 1991, Icarus, 92, 280

Smith, M. D. 2004, Icarus, 167, 148

Summers, M. E., Lieb, B. J., Chapman, E., \& Yung, Y. L. 2002, Geophys. Res. Lett., 29, 2171

Wong, A.-S., Atreya, S. K., \& Encrenaz, T. 2003, J. Geophys. Res. (Planets), 108,5026 